\title{
Symposium review: Mining metagenomic and metatranscriptomic data for clues about microbial metabolic functions in ruminants ${ }^{1}$
}

\author{
Fuyong Li, Andre L. A. Neves, Bibaswan Ghoshal, and Le Luo Guan ${ }^{2}$ \\ Department of Agricultural, Food and Nutritional Science, University of Alberta, Edmonton, Alberta, Canada T6G 2P5
}

\begin{abstract}
Metagenomics and metatranscriptomics can capture the whole genome and transcriptome repertoire of microorganisms through sequencing total DNA/ RNA from various environmental samples, providing both taxonomic and functional information with high resolution. The unique and complex rumen microbial ecosystem is receiving great research attention because the rumen microbiota coevolves with the host and equips ruminants with the ability to convert cellulosic plant materials to high-protein products for human consumption. To date, hundreds to thousands of microbial phylotypes have been identified in the rumen using culture-independent molecular-based approaches, and genomic information of rumen microorganisms is rapidly accumulating through the single genome sequencing. However, functional characteristics of the rumen microbiome have not been well described because there are numerous uncultivable microorganisms in the rumen. The advent of metagenomics and metatranscriptomics along with advanced bioinformatics methods can help us better understand mechanisms of the rumen fermentation, which is vital for improving nutrient utilization and animal productivity. Therefore, in this review, we summarize a general workflow to conduct rumen metagenomics and metatranscriptomics and discuss how the data can be interpreted to be useful information. Moreover, we review recent literatures studying associations between the rumen microbiome and host phenotypes (e.g., feed efficiency and methane emissions) using these approaches, aiming to provide a useful guide to include studying the rumen microbiome as one of the research objectives using these 2 approaches.
\end{abstract}

\footnotetext{
Received June 19, 2017.

Accepted October 27, 2017.

${ }^{1}$ Presented as part of the Growth and Development Symposium: Microbial Endocrinology in Ruminant Growth and Development at the ADSA Annual Meeting, Pittsburgh, Pennsylvania, June 2017.

${ }^{2}$ Corresponding author: lguan@ualberta.ca
}

Key words: rumen, microbiome, microbiota, metagenomics, metatranscriptomics

\section{INTRODUCTION}

The rumen provides anaerobic conditions and redox potentials that favor microbial growth and expression of fiber-degrading enzymes, which allows rumen microorganisms to break down cellulosic plant materials and to meet ruminants' daily energy requirement through producing VFA. Meanwhile, rumen microorganisms are also responsible for producing greenhouse gases [e.g., methane $\left.\left(\mathrm{CH}_{4}\right), \mathrm{CO}_{2}\right]$. It has been suggested that the rumen microbiome is associated with host phenotypes, such as feed efficiency (Hernandez-Sanabria et al., 2010; Shabat et al., 2016), $\mathrm{CH}_{4}$ emissions (Shi et al., 2014; Wallace et al., 2015), milk production (Jami et al., 2014), and ruminal acidosis (Khafipour et al., 2009). Due to their importance, rumen microorganisms have attracted increased attention, and rumen microbiology research has rapidly evolved in the last decade to examine their microbial phylogenetic diversities and functional characteristics.

To date, hundreds to thousands of microbial phylotypes have been identified from various rumen samples using sequencing of marker gene PCR amplicons (amplicon sequencing). In this review, the term microbiota refers to the assemblage of all microorganisms within a microbial community, and the term microbiome refers to the entirety of microbial genomes and transcriptomes from a microbiota, being consistent with the recent definitions from Claesson et al. (2017). It is reported that the rumen microbiota consists of bacteria, archaea, fungi, ciliated protozoa, and phages (with the concentration up to $10^{11}, 10^{9}, 10^{6}, 10^{6}$, and $10^{10}$ per gram of digesta or per milliliter of fluid, respectively; Morgavi et al., 2013). At the same time, genomic information of rumen microorganisms has rapidly accumulated through single genome sequencing, particularly via the recent Hungate 1000 project (http:// www.rmgnetwork.org/hungate1000.html). Based on the genomic information, underlying functions of each sequenced phylotype can be predicted. However, due 
to the lack of available genomes for many important but uncultivable rumen microorganisms (e.g., members belonging to Bacteroidetes), there is still a large gap in the accurate prediction of rumen microbial functional profiles based on the existing genomic information. Therefore, metagenomics and metatranscriptomics represent useful tools for globally cataloging microbial gene and transcript profiles and reflecting overall metabolic functions of rumen microorganisms.

The development of next-generation sequencing (NGS) technologies has accelerated research of the microbiome using metagenomics and metatranscriptomics. Metagenomics and metatranscriptomics are approaches to studying the entirety of genomes (genes) and transcriptomes (transcripts) of a microbial community (Aguiar-Pulido et al., 2016). Initially, these methodologies started with the cloning of environmental DNA and RNA into vectors (e.g., fosmids, bacterial artificial chromosome vectors), followed by sequence-based or function-driven analysis. Nevertheless, the rapid reduction of costs for NGS accelerated the development of those methodologies, and currently they are usually defined as the direct high-throughput shotgun sequencing of total DNA and RNA in environmental samples (also known as shotgun metagenomics and metatranscriptomics). In this review, we discuss a general workflow to conduct rumen metagenomics and metatranscriptomics with the focus on the direct high-throughput shotgun sequencing and summarize implications of these 2 approaches, aiming to provide a useful guide for the efficient application of those 2 approaches to studying the rumen microbiome in the future.

\section{UNDERSTANDING THE MICROBIOME USING SEQUENCING-BASED APPROACHES}

As mentioned above, metagenomics and metatranscriptomics have become powerful and feasible tools for exploring the microbiome of an ecosystem. By using metagenomics and metatranscriptomics, functional potentials (DNA based) and functional activities (RNA based) can be estimated, respectively. The advantage of these approaches is that they can better answer the 2 basic questions "Which members are there?" and "What are they doing?"

\section{"Which Members Are There?"}

To date, culture-independent molecular-based taxonomic analysis of the microbiota highly relies on the sequencing of PCR amplicons of targeted marker genes and transcripts [e.g., $16 \mathrm{~S} \mathrm{rDNA} / \mathrm{rRNA}$ for bacteria and archaea, 18S rDNA/rRNA for protozoa, and internal transcribed spacer (ITS) gene/transcript for fungi], also known as amplicon sequencing. This approach is rapid and low cost; however, the taxonomic assessment of the microbiota can be misleading due to the PCR biases from the primer selection (Hong et al., 2009) and amplification cycling conditions (Huber et al., 2009). In addition, amplicon sequencing has limited potential for discovering new phylotypes because PCR primers are usually designed according to known sequences (Urich et al., 2008; Ross et al., 2012). Recently, it has been reported that metagenomics and metatranscriptomics can be used for the taxonomic assessment of the microbiota, which is less biased and more quantitative than amplicon sequencing (Logares et al., 2014; Li et al., 2016).

\section{"What Are They Doing?"}

For a long time, the study of microbial functions highly relied on pure culture-based approaches, which allow researchers to investigate a specific isolate's metabolic functions and to obtain its single genome or transcriptome. However, culture-based methods are not able to characterize functions of uncultured microorganisms or to elucidate overall functions of all microorganisms within a complex microbiota. Because metagenomics and metatranscriptomics can capture the whole genomic and transcriptomic repertoire for both cultivable and uncultivable microorganisms, they are immensely helpful in the functional prediction.

Until now, the functional repertoires of microbiomes from various ecosystems have been explored using metagenomics and metatranscriptomics, including soil (Urich et al., 2008; Tveit et al., 2014), sea water (Baker et al., 2013; Martínez et al., 2013), the animal gastrointestinal tract (Qin et al., 2010; Franzosa et al., 2014), and the plant rhizosphere (Mendes et al., 2014). However, the number of rumen-related metagenomic and metatranscriptomic studies is still low. The understanding of microbial taxonomic and functional characteristics is vital to link the rumen microbiome to host phenotypes, which can help us develop strategies to optimize rumen microbial fermentation for higher productivity.

\section{A GENERAL WORKFLOW TO CONDUCT RUMEN METAGENOMICS AND METATRANSCRIPTOMICS}

As more and more researchers are interested in applying metagenomics and metatranscriptomics to answer research questions in various ruminant trials, it is necessary to generate high-quality data and have reliable bioinformatics pipelines for these 2 approaches. In this section, we provide an overview of the major 


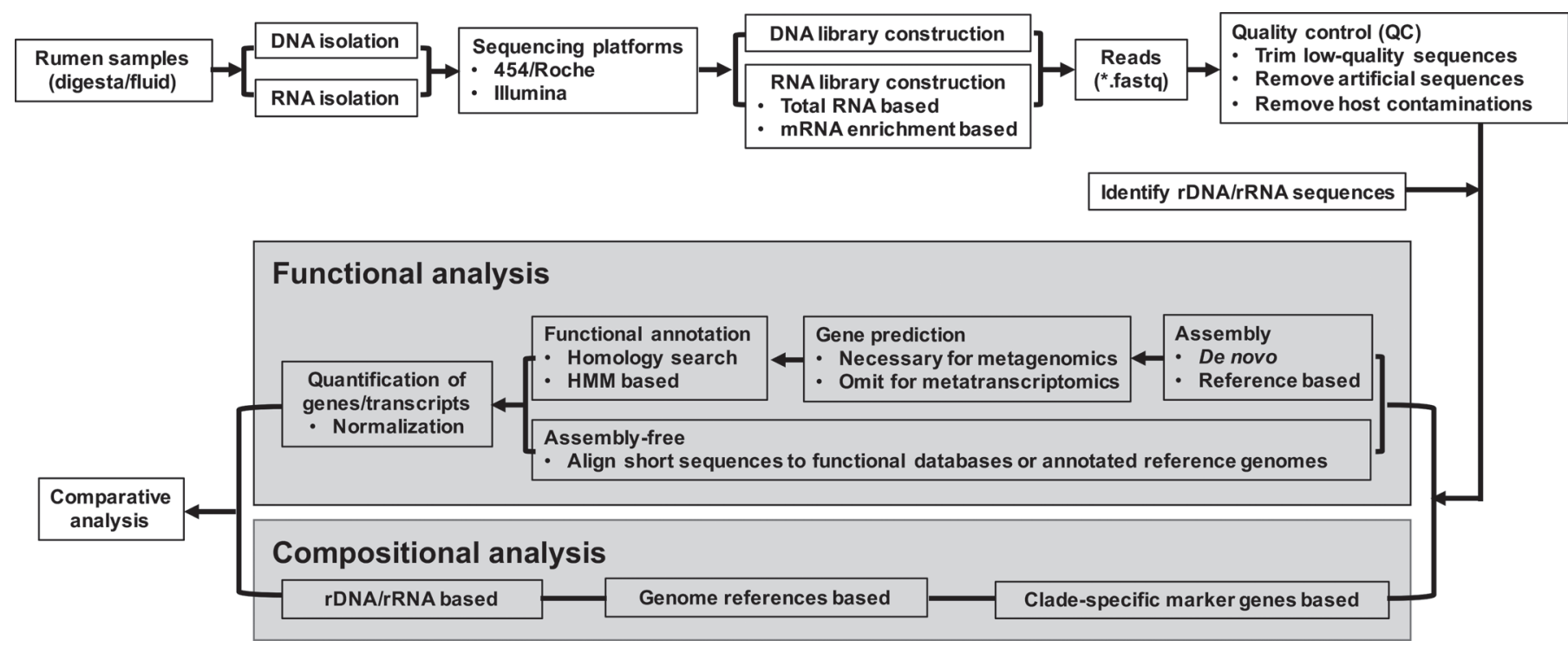

Figure 1. A general workflow to conduct rumen metagenomics and metatranscriptomics. To perform the functional analysis of metatranscriptomic data, it is necessary to discard rRNA sequences and only include putative mRNA in the analysis. HMM = hidden Markov models.

steps of metagenomics and metatranscriptomics (Figure 1), including nucleic acid extraction, sequencing platform selection, library construction, quality control of sequencing outputs, compositional analysis, assembly-based functional analysis, assembly-free functional analysis, and comparative analysis.

\section{DNA and RNA Extraction}

To perform metagenomics and metatranscriptomics, the first step is to isolate high-quality DNA and RNA from rumen samples. Yu and Morrison (2004) and Henderson et al. (2013) compared effects of different DNA extraction methods on rumen samples. Although it is not conclusive which method is universally reliable in all situations, these 2 studies recommended using repeated bead beating plus column or phenol-chloroform and bead beating with filtration kit methods to isolate DNA from rumen samples. Wang et al. (2011) developed an RNA extraction method specifically for rumen samples, which is based on liquid nitrogen grinding of whole ruminal solids and acid guanidinium-phenolchloroform extraction plus column purification. This method was used in the rumen metatranscriptomic study conducted by Qi et al. (2011) and then further optimized by Li et al. (2016). High-quality RNA with an RNA integrity number $>7.0$ can be obtained using these rumen-specific RNA extraction protocols [e.g., Li et al. (2016) obtained an RNA integrity number $>9.0$ for 7 out of 20 rumen digesta samples], which are recommended for further rumen metatranscriptomic studies.

\section{Sequencing Platform Selection and Library Construction}

To date, there are 2 commonly used sequencing platforms for metagenomics and metatranscriptomics: the Roche/454 platform (out of business currently) and the Illumina system (https://www.illumina.com). A few studies have also tried the Pacific Biosciences (PacBio) platform (http://www.pacb.com/; Tsai et al., 2016; Driscoll et al., 2017) and the Ion Torrent platform (https://www.thermofisher.com; Whiteley et al., 2012). Currently, the majority of rumen-related studies rely on the Illumina system; thus, the following sections focus on this system.

For metagenomic and metatranscriptomic library construction, Illumina provides a wide variety of commercial kits. The selection of a kit should be based on research hypotheses and objectives as well as DNA and RNA quality and quantity. For metatranscriptomic studies, before generating libraries, whether the mRNA enrichment is necessary or not should be considered based on research objectives. Because a large fraction of total rumen microbial RNA constitutes rRNA (92.9-96.7\%; Poulsen et al., 2013; Li et al., 2016), the enrichment of mRNA can be conducted to increase the sequencing depth of mRNA to capture more active functional transcripts. For example, Shi et al. (2014) and AlZahal et al. (2017) successfully enriched mRNA from rumen content and fluid samples using commercial kits. However, Tveit et al. (2014) reported that the mRNA enrichment biased the distribution of mRNA, although 
it increased the proportion of mRNA. Hence, Tveit et al. (2014) suggested omitting the mRNA enrichment step and using total RNA for the library construction, which can provide both taxonomic information (estimated based on rRNA) and functional profiles (derived from mRNA) for the active microbiome. With the reduced NGS costs, sequencing total RNA with greater depth became more feasible, which is recommended if researchers aim to generate both compositional and functional profiles using the same metatranscriptomic data set.

\section{Quality Control of Sequencing Outputs}

After obtaining sequencing outputs, crucial steps before further analyses include assessing the output quality and performing quality control (QC), which mainly includes 2 aspects while studying the rumen microbiome. The first aspect of $\mathrm{QC}$ is trimming low-quality bases and residual artificial sequences. These noises decrease the accuracy of taxonomic analysis (Bokulich et al., 2013), disturb sequence mapping, and affect de novo assembly (Sturm et al., 2016). Several programs have been developed for this step, mainly including Trimmomatic (Bolger et al., 2014), PRINSEQ (Schmieder and Edwards, 2011), FASTX-Toolkit (http://hannonlab .cshl.edu/fastx_toolkit/), and NGS QC Toolkit (Patel and Jain, 2012). The main functions of these widely used programs are summarized in Table 1. Among these programs, Trimmomatic is a flexible and efficient preprocessing tool that is specifically designed for Illumina outputs and can generate outcomes superior to, or at least competitive with, other programs such as PRINSEQ and Fastx-Toolkit (Del Fabbro et al., 2013; Bolger et al., 2014). Thus, we recommend using this program for the QC process of Illumina outputs. In addition to the programs listed in Table 1, there are other available options that have been briefly compared by Pandey et al. (2016), to which the reader is referred. In addition, denoising is recommended for $\mathrm{QC}$ of longer sequences generated using Roche/454, PacBio, or Ion Torrent due to their intrinsic errors (error rates of $13 \%$ for PacBio and $1.78 \%$ for Ion Torrent, and high error rates in homopolymers for Roche/454; Metzker, 2010; Quail et al., 2012; Oulas et al., 2015), which usually lead to errors in regards to "unique" identifiers and reduces the clarity for alignments. For the short sequences generated from Illumina, denoising is less required (Oulas et al., 2015), and thus it has been rarely emphasized in any Illumina-based metagenomics and metatranscriptomic studies (Claesson et al., 2017).

The second aspect of $\mathrm{QC}$ is removing host sequences because these sequence contaminations possibly cause misassembly of microbial sequences and lead to erroneous conclusions (Schmieder and Edwards, 2011). In human fecal and milk samples, human DNA sequences reached as high as 64 and $77 \%$ of total metagenome libraries, respectively, suggesting that contamination of

Table 1. Widely used programs for quality control (QC) of sequences

\begin{tabular}{|c|c|c|}
\hline Program & Input & Major QC functions \\
\hline $\begin{array}{l}\text { Trimmomatic } \\
\text { (Bolger et al., 2014) }\end{array}$ & $\begin{array}{l}\text { Single end and paired end } \\
\text { (Illumina) }\end{array}$ & $\begin{array}{l}\text { 1. Cut primer, adapter, and other artificial sequences } \\
\text { 2. Drop low-quality sequences } \\
\text { 3. Trim low-quality bases } \\
\text { 4. Crop sequences to certain length }\end{array}$ \\
\hline $\begin{array}{l}\text { PRINSEQ } \\
\text { (Schmieder and Edwards, 2011) }\end{array}$ & $\begin{array}{l}\text { Single end and paired end } \\
\text { (Illumina, 454/Roche, Ion Torrent) }\end{array}$ & $\begin{array}{l}\text { 1. Perform summary statistics of sequences } \\
\text { 2. Drop low-quality sequences } \\
\text { 3. Trim low-quality bases } \\
\text { 4. Crop sequences to certain length } \\
\text { 5. Filter sequences by GC content }\end{array}$ \\
\hline $\begin{array}{l}\text { NGS QC Toolkit } \\
\text { (Patel and Jain, 2012) }\end{array}$ & $\begin{array}{l}\text { Single end and paired end } \\
\text { (Illumina, Roche/454) }\end{array}$ & $\begin{array}{l}\text { 1. Perform summary statistics of sequences } \\
\text { 2. Cut primer, adapter, and other artificial sequences } \\
\text { 3. Drop low-quality sequences } \\
\text { 4. Crop sequences to certain length } \\
\text { 5. Trim homopolymers }\end{array}$ \\
\hline $\begin{array}{l}\text { FASTX-Toolkit } \\
\text { (http://hannonlab.cshl.edu/fastx_toolkit/) }\end{array}$ & $\begin{array}{l}\text { Single end } \\
\text { (Illumina, 454/Roche, Ion Torrent) }\end{array}$ & $\begin{array}{l}\text { 1. Perform summary statistics of sequences } \\
\text { 2. Cut primer, adapter, and other artificial sequences } \\
\text { 3. Drop low-quality sequences } \\
\text { 4. Trim low-quality bases } \\
\text { 5. Crop sequences to certain length }\end{array}$ \\
\hline $\begin{array}{l}\text { ClinQC } \\
\text { (Pandey et al., 2016) }\end{array}$ & $\begin{array}{l}\text { Single end and paired end } \\
\text { (Illumina, 454/Roche, Ion Torrent, } \\
\text { Sanger) }\end{array}$ & $\begin{array}{l}\text { 1. Perform summary statistics of sequences } \\
\text { 2. Cut primer, adapter, and other artificial sequences } \\
\text { 3. Drop low-quality sequences } \\
\text { 4. Trim low-quality bases }\end{array}$ \\
\hline $\begin{array}{l}\text { SolexaQA } \\
\text { (Cox et al., 2010) }\end{array}$ & $\begin{array}{l}\text { Single end and paired end } \\
\text { (Illumina, 454/Roche, Ion Torrent) }\end{array}$ & $\begin{array}{l}\text { 1. Perform summary statistics of sequences } \\
\text { 2. Drop low-quality sequences } \\
\text { 3. Trim low-quality bases }\end{array}$ \\
\hline
\end{tabular}


host genetic materials is a serious concern for metagenomic studies (Schmieder and Edwards, 2011; Ward et al., 2013). In the rumen metagenome and metatranscriptome, although host DNA and RNA contaminations showed low proportion $(0.43 \%$ in metagenomic data sets and $0.23 \%$ in metatranscriptomic data sets; Shabat et al., 2016; Li and Guan, 2017), checking and discarding them are still recommended. To filter the host (e.g., Bos taurus for cattle and Ovis aries for sheep) sequences, sequences from rumen metagenomic and metatranscriptomic data sets need to be aligned to the host (Bos tauras or Ovis aries) genome, and mapped sequences should be discarded. In previous studies, the MG-RAST (Meyer et al., 2008)-based pipeline and the TopHat2 (Kim et al., 2013)-based approach have been used by Shabat et al. (2016) and Li and Guan (2017) for this step, respectively. MG-RAST is a web-based data analysis pipeline, whereas TopHat2 is a program to map and align sequences to the reference genome using a local computer. Both methods can be used to filter bovine DNA and RNA sequences from rumen metagenomic or metatranscriptomic data sets (Shabat et al., 2016; Li and Guan, 2017). For this step, the algorithms behind these 2 methods were both Bowtie based (Langmead and Salzberg, 2012), and thus the overall outcomes between them should not be significantly different. Researchers can make their own choices based on their available computing sources. If they have a local computer with high calculation capacity, they can run the TopHat2-based pipeline; if they do not have a powerful computer, MG-RAST can be a good choice, but the running time after uploading data to MGRAST is longer than running the analysis locally.

\section{Compositional Analysis}

Here, before detailing how the metagenomic and metatranscriptomic data sets can be used for taxonomic assessment, we highlight the main principles and some drawbacks of profiling the rumen microbiota using amplicon sequencing. To achieve the taxonomic analysis of amplicon sequencing, there are 3 main strategies for defining operational taxonomic unit (OTU): de novo, closed-reference, and open-reference OTU picking (Navas-Molina et al., 2013). Most existing analyses for amplicon sequencing were based on the arbitrary definition of OTU. Sequences are clustered into OTU based on similarity (typically $97 \%$ similarity for $16 \mathrm{~S}$ rDNA sequences), which is called de novo OTU picking (Rideout et al., 2014). Although this 97\% similarity was considered sufficient to delineate species (Koeppel and $\mathrm{Wu}, 2013)$, it is only approximate. Sometimes different species have similar $16 \mathrm{~S}$ rDNA sequences with high identity ( $\geq 99 \%)$, such as Bacillus globisporus and
Bacillus psychrophilus (Fox et al., 1992) as well as Clostridium botulinum and Clostridium sporogenes (RossiTamisier et al., 2015). On the contrary, multiple copies of $16 \mathrm{~S}$ rDNA from a single species may have similarity lower than 97\% (Větrovský and Baldrian, 2013). In addition, the accuracy of the similarity cutoff depends on the choice of different hypervariable regions (Kim et al., 2011). For instance, instead of $97 \%$ similarity, $98 \%$ is more accurate to cluster archaeal species-level OTU when the V4-V7 regions are targeted, whereas $96 \%$ is more appropriate to cluster bacterial species-level OTU when the V1-V4 regions are targeted (Kim et al., 2011). Accordingly, the diversity of the microbiota may not be accurately estimated based on these arbitrarily defined OTU, and analysis at the OTU level may not reflect the "true" and "biological meaningful" microbial composition completely. Therefore, along with the improvement of reference databases, aligning sequences directly to predefined reference sequences (called closed-reference OTU picking; Rideout et al., 2014) is a potential choice to avoid the problem of de novo OTU picking. However, it is notable that closed-reference OTU picking cannot identify novel phylotypes because sequences not matched to references are excluded. Therefore, after closed-reference OTU picking, performing an additional step of de novo OTU picking for those sequences that failed to match to references (called open-reference OTU picking) can solve this problem (Rideout et al., 2014); this is recommended for the taxonomic analysis using amplicon sequencing.

Taxonomic classification of $16 \mathrm{~S}$ sequences usually relied on 4 databases for rumen bacteria: Greengenes (McDonald et al., 2012), SILVA (Quast et al., 2013), RDP (Cole et al., 2014), and NCBI (Federhen, 2012). Although a recent study has compared different taxonomic outcomes of these 4 commonly used bacterial databases (Balvočiūte and Huson, 2017), no study has compared the accuracy and reliability of these databases on assessment of the rumen microbiota. Therefore, it is not conclusive which database is the most suitable for profiling rumen bacteria. Nevertheless, it is worth mentioning that several recent rumen studies, to which the reader is referred, relied on the Greengenes database for taxonomic analyses (Henderson et al., 2015; Wallace et al., 2015; Li and Guan, 2017). To estimate rumen archaeal communities, a rumen-specific archaeal database was first developed by Janssen and Kirs (2008), and later the Rumen and Intestinal Methanogen database (RIM-DB; Seedorf et al., 2014) was developed to achieve species-level taxonomic assignment for rumen archaea. The RIM-DB resulted in more detailed assignments for rumen archaeal sequences than Greengenes and SILVA (Seedorf et al., 2014), which has been applied in recent studies on the rumen microbiota (Hen- 
derson et al., 2015; Seedorf et al., 2015). Therefore, the authors strongly recommend using the RIM-DB to assess rumen archaeal composition for future studies.

In addition to the amplicon sequencing approach mentioned above, taxonomic analysis can be performed based on metagenomic and metatranscriptomic data sets. From metagenomic data sets, the taxonomic profiles can be estimated based on metagenomic 16S rDNA sequences. This strategy was first suggested and tested in marine metagenomes by Logares et al. (2014). Later, Ellison et al. (2014) and Taxis et al. (2015) applied this approach to evaluate rumen microbial composition. Due to the low proportion of $16 \mathrm{~S}$ rDNA among total DNA (0.001-0.12\%; Ellison et al., 2014; Logares et al., 2014), the shortcoming of this approach is that high sequencing depth is required to generate enough 16S rDNA sequences, which dramatically increases the costs. For metatranscriptomic studies, $16 \mathrm{~S}$ rRNA extracted from total RNA sequencing can be used to generate rumen taxonomic profiles and to represent active microbial taxa. This approach was first developed for soil metatranscriptomes by Urich et al. (2008) and then was applied to study the active microbiota in soil (Tveit et al., 2014), hydrothermal vents (Lanzén et al., 2011), and the animal gut (Poulsen et al., 2013; Schwab et al., 2014). In our recent study (Li et al., 2016), a pipeline was developed to taxonomically assess the rumen microbiota (mainly bacterial and archaea) using total RNA-based metatranscriptomics. In this pipeline, regionally enriched reference databases were built for bacteria and archaea and were used for the taxonomic analysis. This approach can prevent the biased taxonomic assessment caused by the short Illumina sequences that have multiple hits when full-length $16 \mathrm{~S}$ rRNA gene databases are used as references. The feasibility and advantages of using this pipeline to assess the rumen microbiota were confirmed when the outcomes of this method were compared with outcomes from $16 \mathrm{~S}$ rDNA and rRNA amplicon sequencing (Li et al., 2016). To perform these rDNA- and rRNA-based taxonomic analyses, rDNA and rRNA sequences should be identified and extracted from metagenomic and metatranscriptomic data sets, which can be achieved using programs such as SortMeRNA (Kopylova et al., 2012), Bowtie2 (Langmead and Salzberg, 2012), and BLAST (Altschul et al., 1990).

Furthermore, other alternative strategies, such as aligning total metagenomic and metatranscriptomic sequences to genome references or clade-specific marker gene databases, are also used to generate the taxonomic profiles, which have been adopted in the various bioinformatics pipelines, including the MG-RAST pipeline (Meyer et al., 2008), Phymm/PhymmBL program (Brady and Salzberg, 2009), and MetaPhlAn software
(Segata et al., 2012). In addition, Andre L. A. Neves (University of Alberta, Edmonton, Alberta, Canada), Fuyong Li (University of Alberta, Edmonton, Alberta, Canada), Bibaswan Ghoshal (University of Alberta, Edmonton, Alberta, Canada), Tim McAllister (Lethbridge Research and Development Centre, Agriculture and Agri-Food Canada, Lethbridge, Alberta, Canada), Le Luo Guan (University of Alberta, Edmonton, Alberta, Canada; unpublished data) recently developed a pipeline to enhance the compositional analysis of rumen metagenomic data sets using Kraken (Wood and Salzberg, 2014) and customized databases. However, it is notable that these strategies rely heavily on the integrity of databases and are probably not efficient for samples from poorly characterized environments (e.g., the rumen).

\section{FUNCTIONAL ANALYSIS}

\section{Assembly-Based Functional Analysis}

Two Assembly Strategies. Assembly of short sequences is expected to reconstruct genomes and transcriptomes of both cultivable and uncultured organisms from sequencing data sets, which can provide valuable information on protein coding genes or operons for the whole microbiome. Despite the impossibility of obtaining complete genome(s) or transcriptome(s) in most cases, assembly is still worth performing to obtain longer contigs (long sequence fragments combined from short sequences) that will increase the sequence annotation accuracy. For metatranscriptomic data, rRNA sequences should be discarded from the data set before the assembly, and only the filtered putative mRNA sequences should be included in the downstream functional analysis. Two strategies can be used for assembly: reference-based assembly and de novo assembly.

The reference-based assembly strategy works only when sequences are from species with known reference genomes or from species closely related to the known reference genomes. If there are insertions, deletions, or copy number variations, these regions cannot be successfully assembled using reference-based approaches (Thomas et al., 2012). Compared with reference-based assembly, de novo assembly reconstructs contigs from sequencing data sets without the aid of genome information. Therefore, considering the complexity of the rumen microbiome and the unavailability of a high number of rumen microbial genomes, the de novo assembly strategy is primarily recommended in rumenrelated metagenomic and metatranscriptomic studies. Currently, most of the existing de novo assemblers have been designed based on the de Bruijn graph, such as Meta-Velvet (Namiki et al., 2012), SOAPdenovo2 
(Luo et al., 2012), Velvet (Zerbino and Birney, 2008), MEGAHIT (Li et al., 2015), and metaSPAdes (Nurk et al., 2017). Until now, the majority of rumen-related metagenomic and metatranscriptomic studies have included the de novo assembly step in the analysis, with only 2 exceptions (Poulsen et al., 2013; AlZahal et al., 2017). It is noticeable that due to conserved genome regions, organism variability, and gene shifts, de novo assembly may bring chimeras and ambiguities into contigs, especially for complex microbial communities (Segata et al., 2013) such as the rumen.

Gene Prediction and Functional Annotation. After assembly of metagenomic sequences, gene prediction and annotation procedures are similar to the framework of the single microbial genome analysis. Contigs are scanned to identify protein coding sequences or open reading frames as well as other components. The gene prediction can be achieved using several gene predictors that are particularly designed for metagenomics, including MetaProdigal (Hyatt et al., 2010), MetaGeneMark (Zhu et al., 2010), and FragGeneScan (Rho et al., 2010). The gene prediction step should be omitted from the metatranscriptomic analysis, and assembled RNA contigs (e.g., transcripts) can be used directly for the annotation. The principle behind the most frequent annotation approach is to assign predicted protein coding sequences, open reading frames, or transcripts against well-characterized databases using homology search based on either nucleotide or translated protein sequences. There are several well-accepted databases for the annotation, such as RefSeq (Pruitt et al., 2012), UniProt (Pundir et al., 2017), KEGG (Kanehisa et al., 2012), eggNOG (Huerta-Cepas et al., 2016), COG/KOG (Tatusov et al., 2003), and SEED (Overbeek et al., 2005). Several programs, including BLAST (Altschul et al., 1990), USEARCH (Edgar, 2010), and DIAMOND (Buchfink et al., 2015), are all available to perform homology detection. In addition to the homology-based approach, the hidden Markov models-based method (Eddy, 2011) can be used to identify protein domains and is more suitable for annotating sequences with low identity to references. The annotation procedure can also be conducted through web-based annotation pipelines, such as MG-RAST (Meyer et al., 2008), WebMGA (Wu et al., 2011), and Galaxy (https://usegalaxy.org/), which combine several of the above-mentioned tools.

Quantification of Predicted Genes and Transcripts. After assembly, sequencing data have only quality characteristics (presence and absence of genes and transcripts) without quantity characteristics (abundance and expressions). To perform the subsequent comparative analysis among treatments or sam- ples, original sequences should be mapped to predicted genes and transcripts to estimate the abundance and expressions using tools such as Bowtie2 (Langmead and Salzberg, 2012), BWA (Li and Durbin, 2010), USEARCH (Edgar, 2010), DIAMOND (Buchfink et al., 2015), or BLAST (Altschul et al., 1990). Outputs from these bioinformatics programs are usually SAM (sequence alignment/map), BAM (binary alignment/ map), or BLAST-format files. To make these outputs adapted for the downstream statistical analysis, they should be further converted to count or abundance files using programs including HUMAnN (Abubucker et al., 2012), MEGAN (Huson et al., 2016), HTSeq (Anders et al., 2015), and SAMtools (Li et al., 2009).

\section{Assembly-Free Functional Analysis}

The assembly-free approach is to align original short sequences (post-QC) to existing functional databases or annotated reference genomes, which is considered an alternative way to estimate functional profiles of metagenomes and metatranscriptomes. For metatranscriptomic data, rRNA sequences should be removed and only putative mRNA sequences should be kept for the analysis. This approach has been used in many metagenomic and metatranscriptomic studies (Franzosa et al., 2014; Sanders et al., 2015; Dubin et al., 2016), but only 2 of them are rumen related (Poulsen et al., 2013; AlZahal et al., 2017). The accuracy of the assembly-free approach highly depends on the reference databases. For well-characterized environments, such as the human gut with $77 \%$ of the taxa cultured and genomes identified (Lagier et al., 2016), this approach is reliable and its outcomes are convincing (Franzosa et al., 2014; Dubin et al., 2016). However, for poorly described environments without complete references, such as the rumen (with $>55 \%$ unclassified and unnamed microbial phylotypes; Henderson et al., 2015), only a low proportion of mRNA sequences could be mapped to the KEGG database ( $\sim 8 \%$; Fuyong Li and Le Luo Guan, Department of Agricultural, Food and Nutritional Science, University of Alberta, Edmonton, Alberta, Canada, unpublished data), and the remaining unmapped sequences have to be ignored for the downstream analysis. This can explain why only $2 \mathrm{ru}-$ men-related meta-omics studies adopted this approach (Poulsen et al., 2013; AlZahal et al., 2017).

MG-RAST (Meyer et al., 2008), HUMAnN (Abubucker et al., 2012), and MEGAN (Huson et al., 2016) are well-accepted programs for conducting the assemblyfree approach. The feasibilities of these programs have been tested, and they have been successfully applied to study microbiomes from many environments, such 
as soil (Urich et al., 2008), the animal gut (Franzosa et al., 2014; Sanders et al., 2015), and the plant rhizosphere (Mendes et al., 2014). Therefore, we suggest that these programs can be applied to study the rumen microbiome if the assembly-free approach is to be used. The Hungate 1000 project will provide a comprehensive rumen-specific database after sequencing around 1,000 genomes of rumen bacteria, archaea, fungi, and ciliate protozoa, which will make the assembly-free approach more feasible for characterization of rumen metagenomes and metatranscriptomes in the near future. To date, the Hungate 1000 project has 437 rumen microbial strains sequenced, and 311 genomes are already available at the Joint Genome Institute (http://genome .jgi.doe.gov).

\section{Comparative Analysis}

In the majority of metagenomic and metatranscriptomic studies, the ultimate goal is to identify differences among treatments or samples at taxonomic and functional levels. An important step before the comparative analysis is to apply the appropriate normalization to all related counts (e.g., genes, transcripts, functional pathways) obtained from previous steps. This is because different samples have different sequencing depths, and genes or transcripts are not of the same length. To normalize different sequencing depths, the counts per million reads is commonly calculated by dividing the count of each gene or transcript by the total number of mapped sequences. The limitation of this approach is that the length variations of genes and transcripts are not accounted for; thus, the approach is suitable for comparing the same functional features between samples but not suitable for comparing different functional features within the same sample. If characterizing the functional profile and comparing different genes and transcripts within the same sample is one of the research objectives, it is necessary to take the length of each gene or transcript into consideration and normalize the gene or transcript counts to reads or fragments per kilobase per million mapped sequences. In addition, because most sequencing data are still not normally distributed after normalization, further data transformation is necessary to make the data more appropriate for frequently used normal-distribution-based statistical methods, such as ANOVA or $t$-test based on arcsine square root transformation (Franzosa et al., 2014). Otherwise, approaches specifically designed for sequencing data should be adopted to perform the comparative analysis, including LEfSe (Segata et al., 2011), DESeq2 (Love et al., 2014), and edgeR (Robinson and Smyth, 2007).

\section{IMPLICATIONS OF METAGENOMICS AND METATRANSCIRPTOMICS TO UNDERSTAND THE RUMEN MICROBIOME}

\section{Characterization of Rumen Microbial Functional Characteristics}

Previous rumen metagenomic and metatranscriptomic studies mainly focused on genes and transcripts encoding enzymes for carbohydrate metabolism, especially enzymes for the degradation of lignocellulosic material that comes from plant cell walls. For instance, Brulc et al. (2009) conducted the first shotgun metagenomicsbased study to screen carbohydrate-active enzymes and identified genes encoding glycoside hydrolases $(\mathrm{n}=35)$, carbohydrate-binding modules $(\mathrm{n}=3)$, carbohydrate esterases $(n=5)$, and pectate lyases $(n=1)$ from the fiber-adherent rumen microorganisms in beef cattle. Later on, shotgun metagenomics was applied to identify biomass-degrading genes in rumen microorganisms of dairy cattle by Hess et al. (2011). In that study, a total of 27,755 carbohydrate-active genes were detected and 90 proteins were expressed; $57 \%$ of them were related to the degradation of cellulosic substrates. Meantime, Qi et al. (2011) performed a metatranscriptomic analysis to estimate functions of rumen eukaryotes in muskoxen and reported the expressions of genes encoding glycoside hydrolases, carbohydrate esterases, pectate lyases, and carbohydrate-binding modules in eukaryotes of the muskoxen rumen. Several follow-up studies have been conducted to detect carbohydrate-degrading enzymes as well as related components in diverse ruminant species using metagenomics and metatranscriptomics (Findley et al., 2011; Dai et al., 2012; Pope et al., 2012; Lim et al., 2013; Gruninger et al., 2014; Lopes et al., 2015; Bensoussan et al., 2017; Comtet-Marre et al., 2017; Jose et al., 2017). These results demonstrate that metagenomics and metatranscriptomics could increase the resolution for microbial functional profiling and capture more microbial enzymes for rumen fermentation.

Recently, metagenomics and metatranscriptomics have been applied to study the rumen virome, whose composition and functions are largely unknown. Berg Miller et al. (2012) conducted the first metagenomicsbased survey of the rumen virome in dairy cattle and reported that the rumen virome was enriched for phages and transposable elements. According to their results, rumen viruses not only had large numbers but also displayed high diversity. Later on, Ross et al. (2013c) obtained 14 putative viral sequence fragments from dairy cattle rumen metagenomes. They found taxonomically variable but functionally conserved rumen viromes 
among individual dairy cows. In addition, Yutin et al. (2015) explored the diversity of virophages and identified a new family of virophages using metagenomics. The findings from the above studies reveal that metagenomics is a powerful tool for discovering novel viruses, which opens a new window for intensively studying functions of the rumen virome and better understanding how viruses interact with other microbial groups (bacteria, archaea, protozoa, and fungi) in the rumen.

Metagenomics and metatranscriptomics have also been used to investigate the rumen bacteria communication pattern via commuquorum-sensing systems (Ghali et al., 2016) as well as functional potentials of the rumen microorganisms to degrade royal demolition explosives ( $\mathrm{Li}$ et al., 2014). Overall, these highlight the potentials of metagenomics and metatranscriptomics to comprehensively discover novel functions and features of the rumen microbiome.

\section{Revealing Linkages Between the Rumen Microbiome and Host Phenotypes}

As one of the main factors to shape the rumen microbiome (Henderson et al., 2015), diet contributes to rumen microbial variations at both taxonomic and functional levels; this has been confirmed using metagenomics (Ellison et al., 2014; Campanaro et al., 2017). Metagenomics was also applied to study the rumen metabolic disorder (e.g., frothy bloat) caused by microbial functional shifts after beef cattle received the high-CP diet (Pitta et al., 2016). As discussed above, metagenomics and metatranscriptomics have been mainly used to explore microbial functional characteristics and to discover novel features of the rumen microbiome itself but seldom have been applied to link the rumen microbiome to host phenotypes. Until now, only a handful of reports have appeared to associate rumen metagenomes or metatranscriptomes to host performances, such as feed efficiency and $\mathrm{CH}_{4}$ emissions.

Feed Efficiency. Until now, 2 significant studies have linked the rumen metagenome and metatranscriptome to cattle feed efficiency (Shabat et al., 2016; Li and Guan, 2017). Applying metagenomics to rumen samples from dairy cows, Shabat et al. (2016) revealed that more efficient individuals possess lower diverse rumen microbiomes at both species and gene levels, and the prediction of feed efficiency using genes of rumen microbiomes can reach up to $91 \%$ accuracy. In the meantime, they found that genes enriched in the efficient group mostly belonged to Megasphaera elsdenii, whereas the similar trend was not observed in the inefficient group. Therefore, they suggested that microbiomes of inefficient cattle might utilize more diverse substrates and produce more diverse products, which either have a negative effect on the host or cannot be efficiently absorbed by the host. From the metatranscriptomic study by Li and Guan (2017), we reported a tentatively higher abundance of 4 active microbial taxa and more enriched differential pathways (30 out of 32) and carbohydrate-active enzymes (11 out of 12) in the inefficient beef cattle. It is notable that both metagenomic and metatranscriptomic studies reported that protein and AA metabolism-related pathways were associated with feed efficiency, and rumen microbiomes in inefficient animals may have higher nitrogen metabolism. These findings suggest that rumen microbiomes of inefficient cattle have more diverse functional potentials (at the DNA level) and activities (at the RNA level) than those of efficient cattle, which contribute to the host feed efficiency variations.

$\mathrm{CH}_{4}$ Emissions. The first attempt to link the rumen metagenome to $\mathrm{CH}_{4}$ emissions was conducted in dairy cows by Ross et al. (2013b), who successfully predicted $\mathrm{CH}_{4}$ yields using metagenomic profiles with an accuracy of prediction of 0.466. Soon afterward, Shi et al. (2014) reported that it was the expressions of methanogenesisrelated genes instead of the abundance of these genes that contributed to the $\mathrm{CH}_{4}$ yield variation in sheep when sheep rumen microbiomes were assessed using metagenomics and metatranscriptomics. Specifically, the $\mathrm{CH}_{4}$ metabolism pathway (ko00680), 3 genes encoding subunits of methyl coenzyme $\mathrm{M}$ reductase, and several genes encoding enzymes for the $\mathrm{CO}_{2} / \mathrm{H}_{2}$ pathway were expressed higher in high $\mathrm{CH}_{4}$ emitters. Therefore, they suggested that reducing expression levels of these genes could be a strategy for future $\mathrm{CH}_{4}$ mitigation. Contrary to that study, Wallace et al. (2015) revealed that genes directly or indirectly involved in methanogenesis were on average 2.7 -fold more abundant in beef cattle with a high $\mathrm{CH}_{4}$ yield based on rumen metagenomics, suggesting that functional characteristics of the rumen microbiome at the DNA level should also be considered the target for future $\mathrm{CH}_{4}$ mitigation. Through combining metagenomics, metatranscriptomics, and amplicon sequencing, Kamke et al. (2016) reported that Sharpea spp. and Megasphaera spp. were more abundant and that genes and transcripts for sugar transport, lactate utilization and production, propionate metabolism, and butyrate metabolism were enriched in sheep with a low $\mathrm{CH}_{4}$ yield. These suggest that more hexoses were first fermented to lactate and then converted to butyrate by Sharpea spp. and Megasphaera spp. through a 2-step process in the rumen of those animals with low $\mathrm{CH}_{4}$ yield. In addition, through comparing microbiomes between yaks and Tibetan sheep from high altitude (low $\mathrm{CH}_{4}$ producers) and cattle and ordinary sheep from low altitude (high $\mathrm{CH}_{4}$ producers) using metagenomics, Zhang et al. (2016) found that ruminants with low $\mathrm{CH}_{4}$ 
emissions had more abundant energy- and carbohydrate-metabolic-related functional categories, whereas high $\mathrm{CH}_{4}$ producers had enriched functions related to methanogenesis (e.g., the $\mathrm{CO}_{2} / \mathrm{H}_{2}$ pathway and the methylotrophic pathway). Overall, these suggest that metagenomics and metatranscriptomics can reveal the potentials (DNA) and activities (RNA) of rumen functions or enzymes involved in methanogenesis, providing future targets for $\mathrm{CH}_{4}$ mitigation in ruminants.

Microbial Responses to Feed Additives to Mitigate $\mathrm{CH}_{4}$. Metagenomics and metatranscriptomics were used to look into effects of feed additives on the rumen microbiome. For example, Ross et al. (2013a) investigated the effect of $2 \mathrm{CH}_{4}$-mitigating feed additives (grapemarc and a combination of lipids and tannin) on the rumen microbiome using metagenomics in dairy cattle. These 2 additives actually altered microbiomes in similar ways, both of which affected 4 second-level KEGG categories (i.e., AA metabolism, carbohydrate metabolism, translation, and biosynthesis of other secondary metabolites). Meanwhile, adding rapeseed oil supplementation to the diet of dairy cows reduced $\mathrm{CH}_{4}$ emissions, and the mechanism of this effect was investigated using rumen metatranscriptomics (Poulsen et al., 2013). After adding rapeseed oil to the diet, the downregulated expressions of several microbial genes of enzymes involved in methylamine-based methanogenesis (including dimethylamine permease, dimethylamine permease methyltransferase corrinoid protein, and trimethylamine:corrinoid methyltransferase), were observed, which was related to the decrease in Methanomassiliicoccales. Denman et al. (2015) studied the $\mathrm{CH}_{4}$ inhibition effect of bromochloromethane (an antimethanogenic compound) on goats via metagenomics. A higher abundance of genes involved in the succinate pathway was detected in rumen microbiomes in response to bromochloromethane; thus, the authors speculated that this pathway could primarily consume $\mathrm{H}_{2}$ when methanogenesis was inhibited. The above findings indicate that metagenomics and metatranscriptomics are power tools for detecting the microbial shifts in response to dietary intervention strategies.

\section{SUMMARY AND CONCLUDING REMARKS}

Here, we reviewed how rumen metagenomics and metatranscriptomics can provide in-depth taxonomic and functional information for the rumen microbiome. The application of these technologies allows us to comprehensively assess rumen microbial functional potentials and activities to better understand mechanisms of the rumen microbial fermentation and how it can be attributed to host phenotypic variations. However, standard procedures and general programs for ru- men metagenomics and metatranscriptomics analysis have not yet been well defined; thus, integrating and comparing outcomes from different studies is still challenging. To meet this challenge, we have described major experimental and computational steps of rumen metagenomics and metatranscriptomics and summarized a general workflow through combining existing rumen metagenomic and metatranscriptomic studies. It is noticeable that among different studies there are many variables, including sampling method and sample source (fluid vs. digesta; living animal vs. slaughtered animal), diet, sequencing platform (Illumina vs. Roche/454), sequencing depth, reference database, bioinformatics pipelines, and data analysis pipelines. Although recommendations have been made for major steps of rumen metagenomic and metatranscriptomic data analysis, it is worth mentioning that those recommendations are not universal for all situations. Carefully selecting algorithms and setting parameters for major steps are key to minimizing the variation of bioinformatic analytic tools and pipelines. In future studies, the choice of algorithms and the parameter settings should be further adjusted according to specific research objectives and data set features. Therefore, it is useful to conduct a meta-analysis through combining and comparing all available rumen metagenomic and metatranscriptomic data sets to further optimize the bioinformatic pipelines and to reduce the biases and errors. In addition, advancing the Hungate 1000 project to generate a comprehensive reference database containing genome sequences from rumen bacteria, archaea, fungi, and ciliate protozoa will maximize the utility of metagenomic and metatranscriptomic data sets for better understanding of rumen microbial metabolic functions.

\section{ACKNOWLEDGMENTS}

We thank the Alberta Livestock and Meat Agency (Edmonton, AB, Canada) for their grant support (no. 2013R029R). We also appreciate the financial support to F. Li from the Alberta Innovates-Technology Futures Graduate Student Scholarship and the Natural Sciences and Engineering Research Council of Canada (NSERC; Ottawa, ON, Canada) discovery grant for L. L. Guan.

\section{REFERENCES}

Abubucker, S., N. Segata, J. Goll, A. M. Schubert, J. Izard, B. L. Can-
tarel, B. Rodriguez-Mueller, J. Zucker, M. Thiagarajan, B. Henris-
sat, O. White, S. T. Kelley, B. Methe, P. D. Schloss, D. Gevers, M.
Mitreva, and C. Huttenhower. 2012. Metabolic reconstruction for
metagenomic data and its application to the human microbiome.
PLOS Comput. Biol. 8:e1002358.
Aguiar-Pulido, V., W. Huang, V. Suarez-Ulloa, T. Cickovski, K.
Mathee, and G. Narasimhan. 2016. Metagenomics, metatranscrip- 
tomics, and metabolomics Approaches for microbiome analysis. Evol. Bioinform. Online 12(Suppl. 1):5-16.

Altschul, S. F., W. Gish, W. Miller, E. W. Myers, and D. J. Lipman. 1990. Basic local alignment search tool. J. Mol. Biol. 215:403-410.

AlZahal, O., F. Li, L. L. Guan, N. D. Walker, and B. W. McBride. 2017. Factors influencing ruminal bacterial community diversity and composition and microbial fibrolytic enzyme abundance in lactating dairy cows with a focus on the role of active dry yeast. J. Dairy Sci. 100:4377-4393.

Anders, S., P. T. Pyl, and W. Huber. 2015. HTSeq-A Python framework to work with high-throughput sequencing data. Bioinformatics 31:166-169.

Baker, B. J., C. S. Sheik, C. A. Taylor, S. Jain, A. Bhasi, J. D. Cavalcoli, and G. J. Dick. 2013. Community transcriptomic assembly reveals microbes that contribute to deep-sea carbon and nitrogen cycling. ISME J. 7:1962-1973.

Balvočiūte, M., and D. H. Huson. 2017. SILVA, RDP, Greengenes, NCBI and OTT - How do these taxonomies compare? BMC Genomics 18(Suppl. 2):114.

Bensoussan, L., S. Morais, B. Dassa, N. Friedman, B. Henrissat, V. Lombard, E. A. Bayer, and I. Mizrahi. 2017. Broad phylogeny and functionality of cellulosomal components in the bovine rumen microbiome. Environ. Microbiol. 19:185-197.

Berg Miller, M. E., C. J. Yeoman, N. Chia, S. G. Tringe, F. E. Angly, R. A. Edwards, H. J. Flint, R. Lamed, E. A. Bayer, and B. A. White. 2012. Phage-bacteria relationships and CRISPR elements revealed by a metagenomic survey of the rumen microbiome. Environ. Microbiol. 14:207-227.

Bokulich, N. A., S. Subramanian, J. J. Faith, D. Gevers, J. I. Gordon, R. Knight, D. A. Mills, and J. G. Caporaso. 2013. Quality-filtering vastly improves diversity estimates from Illumina amplicon sequencing. Nat. Methods 10:57-59.

Bolger, A. M., M. Lohse, and B. Usadel. 2014. Trimmomatic: A flexible trimmer for Illumina sequence data. Bioinformatics 30:2114-2120.

Brady, A., and S. L. Salzberg. 2009. Phymm and PhymmBL: Metagenomic phylogenetic classification with interpolated Markov models. Nat. Methods 6:673-676.

Brulc, J. M., D. A. Antonopoulos, M. E. Miller, M. K. Wilson, A. C. Yannarell, E. A. Dinsdale, R. E. Edwards, E. D. Frank, J. B. Emerson, P. Wacklin, P. M. Coutinho, B. Henrissat, K. E. Nelson, and B. A. White. 2009. Gene-centric metagenomics of the fiber-adherent bovine rumen microbiome reveals forage specific glycoside hydrolases. Proc. Natl. Acad. Sci. USA 106:1948-1953.

Buchfink, B., C. Xie, and D. H. Huson. 2015. Fast and sensitive protein alignment using DIAMOND. Nat. Methods 12:59-60.

Campanaro, S., L. Treu, M. Cattani, P. G. Kougias, V. Vendramin, S. Schiavon, F. Tagliapietra, A. Giacomini, and V. Corich. 2017. In vitro fermentation of key dietary compounds with rumen fluid: A genome-centric perspective. Sci. Total Environ. 584-585:683-691.

Claesson, M. J., A. G. Clooney, and P. W. O'Toole. 2017. A clinician's guide to microbiome analysis. Nat. Rev. Gastroenterol. Hepatol. 14:585-595.

Cole, J. R., Q. Wang, J. A. Fish, B. Chai, D. M. McGarrell, Y. Sun, C. T. Brown, A. Porras-Alfaro, C. R. Kuske, and J. M. Tiedje. 2014. Ribosomal Database Project: Data and tools for high throughput rRNA analysis. Nucleic Acids Res. 42:D633-D642.

Comtet-Marre, S., N. Parisot, P. Lepercq, F. Chaucheyras-Durand, P. Mosoni, E. Peyretaillade, A. R. Bayat, K. J. Shingfield, P. Peyret, and E. Forano. 2017. Metatranscriptomics reveals the active bacterial and eukaryotic fibrolytic communities in the rumen of dairy cow fed a mixed diet. Front. Microbiol. 8:67.

Cox, M. P., D. A. Peterson, and P. J. Biggs. 2010. SolexaQA: At-aglance quality assessment of Illumina second-generation sequencing data. BMC Bioinformatics 11:485.

Dai, X., Y. Zhu, Y. Luo, L. Song, D. Liu, L. Liu, F. Chen, M. Wang, J. Li, X. Zeng, Z. Dong, S. Hu, L. Li, J. Xu, L. Huang, and X. Dong. 2012. Metagenomic insights into the fibrolytic microbiome in yak rumen. PLoS One 7:e40430.

Del Fabbro, C., S. Scalabrin, M. Morgante, and F. M. Giorgi. 2013. An extensive evaluation of read trimming effects on Illumina NGS data analysis. PLoS One 8:e85024.
Denman, S. E., G. Martinez Fernandez, T. Shinkai, M. Mitsumori, and C. S. McSweeney. 2015. Metagenomic analysis of the rumen microbial community following inhibition of methane formation by a halogenated methane analog. Front. Microbiol. 6:1087.

Driscoll, C. B., T. G. Otten, N. M. Brown, and T. W. Dreher. 2017. Towards long-read metagenomics: Complete assembly of three novel genomes from bacteria dependent on a diazotrophic cyanobacterium in a freshwater lake co-culture. Stand. Genomic Sci. 12:9.

Dubin, K., M. K. Callahan, B. Ren, R. Khanin, A. Viale, L. Ling, D. No, A. Gobourne, E. Littmann, C. Huttenhower, E. G. Pamer, and J. D. Wolchok. 2016. Intestinal microbiome analyses identify melanoma patients at risk for checkpoint-blockade-induced colitis. Nat. Commun. 7:10391.

Eddy, S. R. 2011. Accelerated profile HMM searches. PLOS Comput. Biol. 7:e1002195.

Edgar, R. C. 2010. Search and clustering orders of magnitude faster than BLAST. Bioinformatics 26:2460-2461.

Ellison, M. J., G. C. Conant, R. R. Cockrum, K. J. Austin, H. Truong, M. Becchi, W. R. Lamberson, and K. M. Cammack. 2014. Diet alters both the structure and taxonomy of the ovine gut microbial ecosystem. DNA Res. 21:115-125.

Federhen, S. 2012. The NCBI Taxonomy database. Nucleic Acids Res. 40:D136-D143.

Findley, S. D., M. R. Mormile, A. Sommer-Hurley, X. C. Zhang, P. Tipton, K. Arnett, J. H. Porter, M. Kerley, and G. Stacey. 2011. Activity-based metagenomic screening and biochemical characterization of bovine ruminal protozoan glycoside hydrolases. Appl. Environ. Microbiol. 77:8106-8113.

Fox, G. E., J. D. Wisotzkey, and P. Jurtshuk Jr.. 1992. How close is close: 16S rRNA sequence identity may not be sufficient to guarantee species identity. Int. J. Syst. Bacteriol. 42:166-170.

Franzosa, E. A., X. C. Morgan, N. Segata, L. Waldron, J. Reyes, A. M. Earl, G. Giannoukos, M. R. Boylan, D. Ciulla, D. Gevers, J. Izard, W. S. Garrett, A. T. Chan, and C. Huttenhower. 2014. Relating the metatranscriptome and metagenome of the human gut. Proc. Natl. Acad. Sci. USA 111:E2329-E2338.

Ghali, I., T. Shinkai, and M. Mitsumori. 2016. Mining of luxS genes from rumen microbial consortia by metagenomic and metatranscriptomic approaches. Anim. Sci. J. 87:666-673.

Gruninger, R. J., X. Gong, R. J. Forster, and T. A. McAllister. 2014. Biochemical and kinetic characterization of the multifunctional beta-glucosidase/beta-xylosidase/alpha-arabinosidase, Bgxa1. Appl. Microbiol. Biotechnol. 98:3003-3012.

Henderson, G., F. Cox, S. Ganesh, A. Jonker, W. Young, and P. H. Janssen. 2015. Rumen microbial community composition varies with diet and host, but a core microbiome is found across a wide geographical range. Sci. Rep. 5:14567.

Henderson, G., F. Cox, S. Kittelmann, V. H. Miri, M. Zethof, S. J. Noel, G. C. Waghorn, and P. H. Janssen. 2013. Effect of DNA extraction methods and sampling techniques on the apparent structure of cow and sheep rumen microbial communities. PLoS One 8:e74787.

Hernandez-Sanabria, E., L. L. Guan, L. A. Goonewardene, M. Li, D. F. Mujibi, P. Stothard, S. S. Moore, and M. C. Leon-Quintero. 2010. Correlation of particular bacterial PCR-denaturing gradient gel electrophoresis patterns with bovine ruminal fermentation parameters and feed efficiency traits. Appl. Environ. Microbiol. 76:6338-6350.

Hess, M., A. Sczyrba, R. Egan, T. W. Kim, H. Chokhawala, G. Schroth, S. Luo, D. S. Clark, F. Chen, T. Zhang, R. I. Mackie, L. A. Pennacchio, S. G. Tringe, A. Visel, T. Woyke, Z. Wang, and E. M. Rubin. 2011. Metagenomic discovery of biomass-degrading genes and genomes from cow rumen. Science 331:463-467.

Hong, S., J. Bunge, C. Leslin, S. Jeon, and S. S. Epstein. 2009. Polymerase chain reaction primers miss half of rRNA microbial diversity. ISME J. 3:1365-1373.

Huber, J. A., H. G. Morrison, S. M. Huse, P. R. Neal, M. L. Sogin, and D. B. Mark Welch. 2009. Effect of PCR amplicon size on assessments of clone library microbial diversity and community structure. Environ. Microbiol. 11:1292-1302. 
Huerta-Cepas, J., D. Szklarczyk, K. Forslund, H. Cook, D. Heller, M. C. Walter, T. Rattei, D. R. Mende, S. Sunagawa, M. Kuhn, L. J. Jensen, C. von Mering, and P. Bork. 2016. eggNOG 4.5: A hierarchical orthology framework with improved functional annotations for eukaryotic, prokaryotic and viral sequences. Nucleic Acids Res. 44(D1):D286-D293.

Huson, D. H., S. Beier, I. Flade, A. Gorska, M. El-Hadidi, S. Mitra, H. J. Ruscheweyh, and R. Tappu. 2016. MEGAN Community Edition-Interactive exploration and analysis of large-scale microbiome sequencing data. PLOS Comput. Biol. 12:e1004957.

Hyatt, D., P. F. LoCascio, L. J. Hauser, and E. C. Uberbacher. 2012. Gene and translation initiation site prediction in metagenomic sequences. Bioinformatics 28:2223-2230.

Jami, E., B. A. White, and I. Mizrahi. 2014. Potential role of the bovine rumen microbiome in modulating milk composition and feed efficiency. PLoS One 9:e85423.

Janssen, P. H., and M. Kirs. 2008. Structure of the archaeal community of the rumen. Appl. Environ. Microbiol. 74:3619-3625.

Jose, V. L., R. P. More, T. Appoothy, and A. S. Arun. 2017. In depth analysis of rumen microbial and carbohydrate-active enzymes profile in Indian crossbred cattle. Syst. Appl. Microbiol. 40:160-170.

Kamke, J., S. Kittelmann, P. Soni, Y. Li, M. Tavendale, S. Ganesh, P. H. Janssen, W. Shi, J. Froula, E. M. Rubin, and G. T. Attwood 2016. Rumen metagenome and metatranscriptome analyses of low methane yield sheep reveals a Sharpea-enriched microbiome characterised by lactic acid formation and utilisation. Microbiome 4:56.

Kanehisa, M., S. Goto, Y. Sato, M. Furumichi, and M. Tanabe. 2012 KEGG for integration and interpretation of large-scale molecular data sets. Nucleic Acids Res. 40:D109-D114.

Khafipour, E., S. Li, J. C. Plaizier, and D. O. Krause. 2009. Rumen microbiome composition determined using two nutritional models of subacute ruminal acidosis. Appl. Environ. Microbiol. 75:7115-7124.

Kim, D., G. Pertea, C. Trapnell, H. Pimentel, R. Kelley, and S. L. Salzberg. 2013. TopHat2: Accurate alignment of transcriptomes in the presence of insertions, deletions and gene fusions. Genome Biol. 14:R36.

Kim, M., M. Morrison, and Z. Yu. 2011. Evaluation of different partial $16 \mathrm{~S}$ rRNA gene sequence regions for phylogenetic analysis of microbiomes. J. Microbiol. Methods 84:81-87.

Koeppel, A. F., and M. Wu. 2013. Surprisingly extensive mixed phylogenetic and ecological signals among bacterial operational taxonomic units. Nucleic Acids Res. 41:5175-5188.

Kopylova, E., L. Noe, and H. Touzet. 2012. SortMeRNA: Fast and accurate filtering of ribosomal RNAs in metatranscriptomic data. Bioinformatics 28:3211-3217.

Lagier, J. C., S. Khelaifia, M. T. Alou, S. Ndongo, N. Dione, P. Hugon, A. Caputo, F. Cadoret, S. I. Traore, E. H. Seck, G. Dubourg, G. Durand, G. Mourembou, E. Guilhot, A. Togo, S. Bellali, D. Bachar, N. Cassir, F. Bittar, J. Delerce, M. Mailhe, D. Ricaboni, M. Bilen, N. P. Dangui Nieko, N. M. Dia Badiane, C. Valles, D. Mouelhi, K. Diop, M. Million, D. Musso, J. Abrahao, E. I. Azhar, F. Bibi, M. Yasir, A. Diallo, C. Sokhna, F. Djossou, V. Vitton, C. Robert, J. M. Rolain, B. La Scola, P. E. Fournier, A. Levasseur, and D. Raoult. 2016. Culture of previously uncultured members of the human gut microbiota by culturomics. Nat. Microbiol. 1:16203.

Langmead, B., and S. L. Salzberg. 2012. Fast gapped-read alignment with Bowtie 2. Nat. Methods 9:357-359.

Lanzén, A., S. L. Jorgensen, M. M. Bengtsson, I. Jonassen, L. Ovreas, and T. Urich. 2011. Exploring the composition and diversity of microbial communities at the Jan Mayen hydrothermal vent field using RNA and DNA. FEMS Microbiol. Ecol. 77:577-589.

Li, D., C. M. Liu, R. Luo, K. Sadakane, and T. W. Lam. 2015. MEGAHIT: An ultra-fast single-node solution for large and complex metagenomics assembly via succinct de Bruijn graph. Bioinformatics 31:1674-1676.

Li, F., and L. L. Guan. 2017. Metatranscriptomic profiling reveals linkages between the active rumen microbiome and feed efficiency in beef cattle. Appl. Environ. Microbiol. 83:e00061-17.

Li, F., G. Henderson, X. Sun, F. Cox, P. H. Janssen, and L. L. Guan 2016. Taxonomic assessment of rumen microbiota using total RNA and targeted amplicon sequencing approaches. Front. Microbiol $7: 987$.

Li, H., and R. Durbin. 2010. Fast and accurate long-read alignment with Burrows-Wheeler transform. Bioinformatics 26:589-595.

Li, H., B. Handsaker, A. Wysoker, T. Fennell, J. Ruan, N. Homer, G. Marth, G. Abecasis, and R. Durbin. 2009. The Sequence Alignment/Map format and SAMtools. Bioinformatics 25:2078-2079.

Li, R. W., J. G. Giarrizzo, S. Wu, W. Li, J. M. Duringer, and A. M. Craig. 2014. Metagenomic insights into the RDX-degrading potential of the ovine rumen microbiome. PLoS One 9:e110505.

Lim, S., J. Seo, H. Choi, D. Yoon, J. Nam, H. Kim, S. Cho, and J. Chang. 2013. Metagenome analysis of protein domain collocation within cellulase genes of goat rumen microbes. Asian-australas. J. Anim. Sci. 26:1144-1151.

Logares, R., S. Sunagawa, G. Salazar, F. M. Cornejo-Castillo, I. Ferrera, H. Sarmento, P. Hingamp, H. Ogata, C. de Vargas, G. LimaMendez, J. Raes, J. Poulain, O. Jaillon, P. Wincker, S. KandelsLewis, E. Karsenti, P. Bork, and S. G. Acinas. 2014. Metagenomic $16 \mathrm{~S}$ rDNA Illumina tags are a powerful alternative to amplicon sequencing to explore diversity and structure of microbial communities. Environ. Microbiol. 16:2659-2671.

Lopes, L. D., A. O. de Souza Lima, R. G. Taketani, P. Darias, L. R. da Silva, E. M. Romagnoli, H. Louvandini, A. L. Abdalla, and R. Mendes. 2015. Exploring the sheep rumen microbiome for carbohydrate-active enzymes. Antonie van Leeuwenhoek 108:15-30.

Love, M. I., W. Huber, and S. Anders. 2014. Moderated estimation of fold change and dispersion for RNA-seq data with DESeq2. Genome Biol. 15:550.

Luo, R., B. Liu, Y. Xie, Z. Li, W. Huang, J. Yuan, G. He, Y. Chen, Q. Pan, Y. Liu, J. Tang, G. Wu, H. Zhang, Y. Shi, Y. Liu, C. Yu, B. Wang, Y. Lu, C. Han, D. W. Cheung, S. M. Yiu, S. Peng, Z. Xiaoqian, G. Liu, X. Liao, Y. Li, H. Yang, J. Wang, T. W. Lam, and J. Wang. 2012. SOAPdenovo2: An empirically improved memoryefficient short-read de novo assembler. Gigascience 1:18.

Martínez, A., L. A. Ventouras, S. T. Wilson, D. M. Karl, and E. F. Delong. 2013. Metatranscriptomic and functional metagenomic analysis of methylphosphonate utilization by marine bacteria. Front. Microbiol. 4:340

McDonald, D., M. N. Price, J. Goodrich, E. P. Nawrocki, T. Z. DeSantis, A. Probst, G. L. Andersen, R. Knight, and P. Hugenholtz. 2012. An improved Greengenes taxonomy with explicit ranks for ecological and evolutionary analyses of bacteria and archaea. ISME J. 6:610-618.

Mendes, L. W., E. E. Kuramae, A. A. Navarrete, J. A. van Veen, and S. M. Tsai. 2014. Taxonomical and functional microbial community selection in soybean rhizosphere. ISME J. 8:1577-1587.

Metzker, M. L. 2010. Sequencing technologies-The next generation. Nat. Rev. Genet. 11:31-46.

Meyer, F., D. Paarmann, M. D'Souza, R. Olson, E. M. Glass, M. Kubal, T. Paczian, A. Rodriguez, R. Stevens, A. Wilke, J. Wilkening, and R. A. Edwards. 2008. The metagenomics RAST serverA public resource for the automatic phylogenetic and functional analysis of metagenomes. BMC Bioinformatics 9:386.

Morgavi, D. P., W. J. Kelly, P. H. Janssen, and G. T. Attwood. 2013 Rumen microbial (meta)genomics and its application to ruminant production. Animal 7(Suppl. 1):184-201.

Namiki, T., T. Hachiya, H. Tanaka, and Y. Sakakibara. 2012. MetaVelvet: An extension of Velvet assembler to de novo metagenome assembly from short sequence reads. Nucleic Acids Res. 40:e155.

Navas-Molina, J. A., J. M. Peralta-Sánchez, A. González, P. J. McMurdie, Y. Vázquez-Baeza, Z. Xu, L. K. Ursell, C. Lauber, H. Zhou, S. J. Song, J. Huntley, G. L. Ackermann, D. Berg-Lyons, S. Holmes, J. G. Caporaso, and R. Knight. 2013. Advancing our understanding of the human microbiome using QIIME. Pages 371-444 in Methods in Enzymology. Vol. 531. E. F. DeLong, ed. Academic Press, Cambridge, MA.

Nurk, S., D. Meleshko, A. Korobeynikov, and P. A. Pevzner. 2017 metaSPAdes: A new versatile metagenomic assembler. Genome Res. 27:824-834.

Oulas, A., C. Pavloudi, P. Polymenakou, G. A. Pavlopoulos, N. Papanikolaou, G. Kotoulas, C. Arvanitidis, and I. Iliopoulos. 2015. 
Metagenomics: Tools and insights for analyzing next-generation sequencing data derived from biodiversity studies. Bioinform. Biol. Insights 9:75-88.

Overbeek, R., T. Begley, R. M. Butler, J. V. Choudhuri, H. Y. Chuang, M. Cohoon, V. de Crecy-Lagard, N. Diaz, T. Disz, R. Edwards, M. Fonstein, E. D. Frank, S. Gerdes, E. M. Glass, A. Goesmann, A. Hanson, D. Iwata-Reuyl, R. Jensen, N. Jamshidi, L. Krause, M. Kubal, N. Larsen, B. Linke, A. C. McHardy, F. Meyer, H. Neuweger, G. Olsen, R. Olson, A. Osterman, V. Portnoy, G. D. Pusch, D. A. Rodionov, C. Ruckert, J. Steiner, R. Stevens, I. Thiele, O. Vassieva, Y. Ye, O. Zagnitko, and V. Vonstein. 2005. The subsystems approach to genome annotation and its use in the project to annotate 1000 genomes. Nucleic Acids Res. 33:5691-5702.

Pandey, R. V., S. Pabinger, A. Kriegner, and A. Weinhausel. 2016. ClinQC: A tool for quality control and cleaning of Sanger and NGS data in clinical research. BMC Bioinformatics 17:56.

Patel, R. K., and M. Jain. 2012. NGS QC Toolkit: A toolkit for quality control of next generation sequencing data. PLoS One 7:e30619.

Pitta, D. W., W. E. Pinchak, N. Indugu, B. Vecchiarelli, R. Sinha, and J. D. Fulford. 2016. Metagenomic analysis of the rumen microbiome of steers with wheat-induced frothy bloat. Front. Microbiol. 7:689.

Pope, P. B., A. K. Mackenzie, I. Gregor, W. Smith, M. A. Sundset, A. C. McHardy, M. Morrison, and V. G. Eijsink. 2012. Metagenomics of the Svalbard reindeer rumen microbiome reveals abundance of polysaccharide utilization loci. PLoS One 7:e38571.

Poulsen, M., C. Schwab, B. B. Jensen, R. M. Engberg, A. Spang, N. Canibe, O. Hojberg, G. Milinovich, L. Fragner, C. Schleper, W. Weckwerth, P. Lund, A. Schramm, and T. Urich. 2013. Methylotrophic methanogenic Thermoplasmata implicated in reduced methane emissions from bovine rumen. Nat. Commun. 4:1428.

Pruitt, K. D., T. Tatusova, G. R. Brown, and D. R. Maglott. 2012. NCBI Reference Sequences (RefSeq): Current status, new features and genome annotation policy. Nucleic Acids Res. 40:D130-D135.

Pundir, S., M. J. Martin, and C. O'Donovan. 2017. UniProt protein knowledgebase. Methods Mol. Biol. 1558:41-55.

Qi, M., P. Wang, N. O'Toole, P. S. Barboza, E. Ungerfeld, M. B. Leigh, L. B. Selinger, G. Butler, A. Tsang, T. A. McAllister, and R. J. Forster. 2011. Snapshot of the eukaryotic gene expression in muskoxen rumen-A metatranscriptomic approach. PLoS One 6:e20521.

Qin, J., R. Li, J. Raes, M. Arumugam, K. S. Burgdorf, C. Manichanh, T. Nielsen, N. Pons, F. Levenez, T. Yamada, D. R. Mende, J. Li, J. Xu, S. Li, D. Li, J. Cao, B. Wang, H. Liang, H. Zheng, Y. Xie, J. Tap, P. Lepage, M. Bertalan, J.-M. Batto, T. Hansen, D. Le Paslier, A. Linneberg, H. B. Nielsen, E. Pelletier, P. Renault, T. Sicheritz-Ponten, K. Turner, H. Zhu, C. Yu, S. Li, M. Jian, Y. Zhou, Y. Li, X. Zhang, S. Li, N. Qin, H. Yang, J. Wang, S. Brunak, J. Dore, F. Guarner, K. Kristiansen, O. Pedersen, J. Parkhill, J. Weissenbach, P. Bork, S. D. Ehrlich, and J. Wang. 2010. A human gut microbial gene catalogue established by metagenomic sequencing. Nature 464:59-65.

Quail, M. A., M. Smith, P. Coupland, T. D. Otto, S. R. Harris, T. R. Connor, A. Bertoni, H. P. Swerdlow, and Y. Gu. 2012. A tale of three next generation sequencing platforms: Comparison of Ion Torrent, Pacific Biosciences and Illumina MiSeq sequencers. BMC Genomics 13:341

Quast, C., E. Pruesse, P. Yilmaz, J. Gerken, T. Schweer, P. Yarza, J. Peplies, and F. O. Glockner. 2013. The SILVA ribosomal RNA gene database project: Improved data processing and web-based tools. Nucleic Acids Res. 41:D590-D596.

Rho, M., H. Tang, and Y. Ye. 2010. FragGeneScan: Predicting genes in short and error-prone reads. Nucleic Acids Res. 38:e191.

Rideout, J. R., Y. He, J. A. Navas-Molina, W. A. Walters, L. K. Ursell, S. M. Gibbons, J. Chase, D. McDonald, A. Gonzalez, A. Robbins-Pianka, J. C. Clemente, J. A. Gilbert, S. M. Huse, H. W. Zhou, R. Knight, and J. G. Caporaso. 2014. Subsampled openreference clustering creates consistent, comprehensive OTU definitions and scales to billions of sequences. PeerJ 2:e545.
Robinson, M. D., and G. K. Smyth. 2007. Moderated statistical tests for assessing differences in tag abundance. Bioinformatics 23:28812887.

Ross, E. M., P. J. Moate, C. R. Bath, S. E. Davidson, T. I. Sawbridge, K. M. Guthridge, B. G. Cocks, and B. J. Hayes. 2012. High throughput whole rumen metagenome profiling using untargeted massively parallel sequencing. BMC Genet. 13:53.

Ross, E. M., P. J. Moate, L. Marett, B. G. Cocks, and B. J. Hayes. 2013a. Investigating the effect of two methane-mitigating diets on the rumen microbiome using massively parallel sequencing. J. Dairy Sci. 96:6030-6046.

Ross, E. M., P. J. Moate, L. C. Marett, B. G. Cocks, and B. J. Hayes. 2013b. Metagenomic predictions: From microbiome to complex health and environmental phenotypes in humans and cattle. PLoS One 8:e73056.

Ross, E. M., S. Petrovski, P. J. Moate, and B. J. Hayes. 2013c. Metagenomics of rumen bacteriophage from thirteen lactating dairy cattle. BMC Microbiol. 13:242.

Rossi-Tamisier, M., S. Benamar, D. Raoult, and P. E. Fournier. 2015. Cautionary tale of using $16 \mathrm{~S}$ rRNA gene sequence similarity values in identification of human-associated bacterial species. Int. J. Syst. Evol. Microbiol. 65:1929-1934.

Sanders, J. G., A. C. Beichman, J. Roman, J. J. Scott, D. Emerson, J. J. McCarthy, and P. R. Girguis. 2015. Baleen whales host a unique gut microbiome with similarities to both carnivores and herbivores. Nat. Commun. 6:8285.

Schmieder, R., and R. Edwards. 2011. Quality control and preprocessing of metagenomic datasets. Bioinformatics 27:863-864.

Schwab, C., D. Berry, I. Rauch, I. Rennisch, J. Ramesmayer, E. Hainzl, S. Heider, T. Decker, L. Kenner, M. Muller, B. Strobl, M. Wagner, C. Schleper, A. Loy, and T. Urich. 2014. Longitudinal study of murine microbiota activity and interactions with the host during acute inflammation and recovery. ISME J. 8:1101-1114.

Seedorf, H., S. Kittelmann, G. Henderson, and P. H. Janssen. 2014 RIM-DB: A taxonomic framework for community structure analysis of methanogenic archaea from the rumen and other intestinal environments. PeerJ 2:e494.

Seedorf, H., S. Kittelmann, and P. H. Janssen. 2015. Few highly abundant operational taxonomic units dominate within rumen methanogenic archaeal species in New Zealand sheep and cattle. Appl. Environ. Microbiol. 81:986-995.

Segata, N., D. Boernigen, T. L. Tickle, X. C. Morgan, W. S. Garrett, and C. Huttenhower. 2013. Computational meta'omics for microbial community studies. Mol. Syst. Biol. 9:666.

Segata, N., J. Izard, L. Waldron, D. Gevers, L. Miropolsky, W. S. Garrett, and C. Huttenhower. 2011. Metagenomic biomarker discovery and explanation. Genome Biol. 12:R60.

Segata, N., L. Waldron, A. Ballarini, V. Narasimhan, O. Jousson, and C. Huttenhower. 2012. Metagenomic microbial community profiling using unique clade-specific marker genes. Nat. Methods 9:811-814.

Shabat, S. K., G. Sasson, A. Doron-Faigenboim, T. Durman, S. Yaacoby, M. E. Berg Miller, B. A. White, N. Shterzer, and I. Mizrahi. 2016. Specific microbiome-dependent mechanisms underlie the energy harvest efficiency of ruminants. ISME J. 10:2958-2972.

Shi, W., C. D. Moon, S. C. Leahy, D. Kang, J. Froula, S. Kittelmann, C. Fan, S. Deutsch, D. Gagic, H. Seedorf, W. J. Kelly, R. Atua C. Sang, P. Soni, D. Li, C. S. Pinares-Patino, J. C. McEwan, P. H. Janssen, F. Chen, A. Visel, Z. Wang, G. T. Attwood, and E. M. Rubin. 2014. Methane yield phenotypes linked to differential gene expression in the sheep rumen microbiome. Genome Res. 24:1517-1525.

Sturm, M., C. Schroeder, and P. Bauer. 2016. SeqPurge: Highly-sensitive adapter trimming for paired-end NGS data. BMC Bioinformatics 17:208

Tatusov, R. L., N. D. Fedorova, J. D. Jackson, A. R. Jacobs, B. Kiryutin, E. V. Koonin, D. M. Krylov, R. Mazumder, S. L. Mekhedov, A. N. Nikolskava, B. S. Rao, S. Smirnov, A. V. Sverdlov, S. Vasudevan, Y. I. Wolf, J. J. Yin, and D. A. Natale. 2003. The COG 
database: An updated version includes eukaryotes. BMC Bioinformatics $4: 41$

Taxis, T. M., S. Wolff, S. J. Gregg, N. O. Minton, C. Zhang, J. Dai, R. D. Schnabel, J. F. Taylor, M. S. Kerley, J. C. Pires, W. R. Lamberson, and G. C. Conant. 2015. The players may change but the game remains: Network analyses of ruminal microbiomes suggest taxonomic differences mask functional similarity. Nucleic Acids Res. 43:9600-9612.

Thomas, T., J. Gilbert, and F. Meyer. 2012. Metagenomics-A guide from sampling to data analysis. Microb. Inform. Exp. 2:3.

Tsai, Y. C., S. Conlan, C. Deming, J. A. Segre, H. H. Kong, J. Korlach, and J. Oh. 2016. Resolving the complexity of human skin metagenomes using single-molecule sequencing. MBio 7:e01948-15.

Tveit, A. T., T. Urich, and M. M. Svenning. 2014. Metatranscriptomic analysis of arctic peat soil microbiota. Appl. Environ. Microbiol. 80:5761-5772.

Urich, T., A. Lanzen, J. Qi, D. H. Huson, C. Schleper, and S. C. Schuster. 2008. Simultaneous assessment of soil microbial community structure and function through analysis of the meta-transcriptome. PLoS One 3:e2527.

Větrovský, T., and P. Baldrian. 2013. The variability of the 16S rRNA gene in bacterial genomes and its consequences for bacterial community analyses. PLoS One 8:e57923.

Wallace, R. J., J. A. Rooke, N. McKain, C. A. Duthie, J. J. Hyslop, D. W. Ross, A. Waterhouse, M. Watson, and R. Roehe. 2015. The rumen microbial metagenome associated with high methane production in cattle. BMC Genomics 16:839.

Wang, P., M. Qi, P. Barboza, M. B. Leigh, E. Ungerfeld, L. B. Selinger, T. A. McAllister, and R. J. Forster. 2011. Isolation of high-quality total RNA from rumen anaerobic bacteria and fungi, and subsequent detection of glycoside hydrolases. Can. J. Microbiol. 57:590-598.

Ward, T. L., S. Hosid, I. Ioshikhes, and I. Altosaar. 2013. Human milk metagenome: A functional capacity analysis. BMC Microbiol. $13: 116$.

Whiteley, A. S., S. Jenkins, I. Waite, N. Kresoje, H. Payne, B. Mullan, R. Allcock, and A. O'Donnell. 2012. Microbial 16S rRNA Ion Tag and community metagenome sequencing using the Ion Torrent (PGM) Platform. J. Microbiol. Methods 91:80-88.

Wood, D. E., and S. L. Salzberg. 2014. Kraken: Ultrafast metagenomic sequence classification using exact alignments. Genome Biol. 15:R46.

Wu, S., Z. Zhu, L. Fu, B. Niu, and W. Li. 2011. WebMGA: A customizable web server for fast metagenomic sequence analysis. BMC Genomics 12:444.

Yu, Z., and M. Morrison. 2004. Improved extraction of PCR-quality community DNA from digesta and fecal samples. Biotechniques $36: 808-812$.

Yutin, N., V. V. Kapitonov, and E. V. Koonin. 2015. A new family of hybrid virophages from an animal gut metagenome. Biol. Direct 10:19.

Zerbino, D. R., and E. Birney. 2008. Velvet: Algorithms for de novo short read assembly using de Bruijn graphs. Genome Res. 18:821829.

Zhang, Z., D. Xu, L. Wang, J. Hao, J. Wang, X. Zhou, W. Wang, Q. Qiu, X. Huang, J. Zhou, R. Long, F. Zhao, and P. Shi. 2016. Convergent evolution of rumen microbiomes in high-altitude mammals. Curr. Biol. 26:1873-1879.

Zhu, W., A. Lomsadze, and M. Borodovsky. 2010. Ab initio gene identification in metagenomic sequences. Nucleic Acids Res. 38:e132. 